\title{
Fisiología del cultivo de yuca en el bosque seco tropical de Sucre - Colombia
}

\section{Physiology of cassava cultivation in the tropical dry forest of Sucre - Colombia}

\author{
Joseth A. Santos*1, Lainer Narváez², Saula M. Salcedo³, Alba N. Acevedo ${ }^{4}$, \\ Luis C. Mercado ${ }^{5}$, Jairo G. Salcedo ${ }^{6}$
}

Recibido para publicación: Mayo 14 de 2018 - Aceptado para publicación: Noviembre 23 de 2018

\begin{abstract}
RESUMEN
La especie M. esculenta presenta gran variabilidad genética que dificulta su estudio debido a que debe ser evaluada no solo por su comportamiento varietal sino también por su respuesta al ambiente donde se produce. Para esta investigación se evaluó el comportamiento fisiológico de yuca en tres de las variedades más cultivadas en el departamento de Sucre: MCOL 2066 (Chirosa: uso industrial y comestible), MCOL 2215 (Venezolana: comestible) y M-Tai 8 (M-Tai: uso industrial), bajo la oferta ambiental del bosque seco tropical, se utilizó un diseño en bloques completos al azar con tres repeticiones. Se evaluó altura de planta (AP), diámetro de tallo (DT), número de hojas $(\mathrm{NH})$, diámetro de raíces tuberosas (DR), fotosíntesis $(P N)$, conductancia estomática $(G S)$, transpiración $(E)$ acumulación y distribución de masa seca e índice de cosecha (IC). Las evaluaciones se llevaron a cabo a los 90, 270, 315 y 360 días después de la siembra (DDS). La variedad M-Tai presentó los resultados más favorables un crecimiento y desarrollo más precoz en los primeros estadios del cultivo (90 DDS) expresado en un mayor porte y mayor acumulación de biomasa con una media de 198,51 gr de materia seca. En la fecha de cosecha 270, 315 y 360 DDS no se observó diferencias entre variables de intercambio gaseoso y acumulación y distribución de biomasa, presentando valores promedios de $130 \mathrm{mmol}$ $\mathrm{H}_{2} \mathrm{O} \mathrm{m}^{-2} \mathrm{~s}^{-1}, 17,87 \mu$ moles $\mathrm{CO}_{2} \mathrm{~m}^{-2} \mathrm{~s}^{-1}$ y $1,99 \mathrm{mmol} \mathrm{H}_{2} \mathrm{O}$ $\mathrm{mm}^{-2} \mathrm{~s}^{-1}$ para GS, PN y $\mathrm{E}$ respectivamente, y con valores alrededor de un $55 \%$ para IC.
\end{abstract}

Palabras clave: Manihot esculenta Crantz, Fenotipo, intercambio gaseoso, morfología.

\begin{abstract}
The species M. esculenta presents great genetic variability that makes its study difficult because it must be evaluated not only for its varietal behavior but also for its response to the environment where it occurs. For this research, the physiological behavior of cassava was evaluatedin three of the most cultivated varieties in the department of Sucre: MCOL 2066 (Chirosa: industrial and edible use), MCOL 2215 (Venezolana: edible use) and M- Tai 8 (M-Tai: industrial use), under the environmental offer of the tropical dry forest, a randomized complete block design with three replications was used. Plant height (AP), stem diameter (DT), number of leaves $(\mathrm{NH})$, diameter of tuberous roots (DR), photosynthesis (PN), stomatal conductance $(\mathrm{GS})$, transpiration $(\mathrm{E})$, accumulation and distribution were evaluated. dry mass and harvest index (Cl). The evaluations were carried out at 90, 270, 315 and 360 days after sowing (DDS). The M-Tai variety presented the most favorable results, an earlier growth and development in the first stages of the crop (90 DDS) expressed in a larger size and greater accumulation of biomass with an average of $198.51 \mathrm{gr}$ of dry matter. At crop date 270, 315 and 360 DDS no differences were observed between variables of gas exchange and biomass accumulation and distribution, presenting average values of $130 \mathrm{mmol} \mathrm{H}_{2} \mathrm{O} \mathrm{m}^{-2} \mathrm{~s}^{-1}, 17.87$ moles $\mathrm{CO}_{2} \mathrm{~m}^{-2} \mathrm{~s}^{-1}$ and $1.99 \mathrm{mmol} \mathrm{H}_{2} \mathrm{O} \mathrm{m}^{-2} \mathrm{~s}^{-1}$ for GS, PN and E respectively, and with values around $55 \%$ for IC.
\end{abstract}

Key words: Manihot esculenta Crantz, Phenotype, gaseous exchange, morphology.

\footnotetext{
${ }^{1}$ Ingeniero Agrícola, investigador PADES, Universidad de Sucre, Sincelejo, Colombia, Calle 25 Bis 1\#9b-19 barrio 6 de febrero, celular: 3005957625, josethalfonzo29@gmail.com ORCID: https://orcid.org/0000-0002-0560-0529.

${ }^{2}$ M.Sc Ciencias Agronómicas, investigador master PADES, Universidad de Sucre, Sincelejo, Colombia, ORCID: https://orcid.org/00000001-7242-4185

${ }^{3}$ M.Sc Ciencias Agronómicas, investigador master PADES, Universidad de Sucre, Sincelejo, Colombia, ORCID: https://orcid.org/00000002-3468-2818

${ }^{4}$ M.Sc Ciencias Agronómicas, investigador master PADES, Universidad de Sucre, Sincelejo, Colombia, ORCID: https://orcid.org/00000001-8535-2360

${ }^{5}$ M.Sc Ciencias Agronómicas, investigador master PADES, Universidad de Sucre, Sincelejo, Colombia, ORCID: https://orcid.org/00000002-8972-5985

6 PhD Ingeniería química, director científico PADES, Universidad de Sucre, Sincelejo, Colombia, ORCID: https://orcid.org/0000-00029901-9793
} 


\section{INTRODUCCIÓN}

La yuca (Manihot esculenta Crantz) pertenece a la familia Euphorbiaceae, es la cuarta fuente de calorías para alrededor de 500 millones de personas, después del arroz, el azúcar y el maíz (Suarez y Mederos, 2011). Es cultivada en América Latina, África y Asia; se encuentra entre los diez principales cultivos en el mundo con aproximadamente 277 millones de toneladas producidas para el 2016, de esos solo 28 millones de toneladas son cosechadas en América del sur (Faostat, 2016). Es el alimento básico en 105 países, incluido Colombia, donde la yuca es el tercer producto de mayor producción, después de la caña de azúcar y plátano; con 2 millones de toneladas y 187 mil hectáreas sembradas con un rendimiento de $11 \mathrm{t} \mathrm{ha}^{-1}$ (Agronet, 2016).

Es también, la fuente de almidón más barata que existe en el mundo y es utilizada en más de 300 productos industriales (FAO, 2008). Si se compara con otras raíces y tubérculos (yuca, papa, camote y ñame) la yuca es la que presentará la mayor tasa de crecimiento de consumo anual hasta el 2020, con 1,9\% y el segundo lugar en término de producción de forraje, con 0,95\% (Rivera et al., 2012) La yuca es un alimento básico en muchas regiones, principalmente en el sector rural más pobre donde se registran los mayores índices de consumo. El $80 \%$ de la yuca que se produce en Colombia se destina al consumo directo, y el $20 \%$ restante se procesa, La producción del departamento de Sucre para el año 2016 representó el $4,32 \%$ de la producción total a nivel nacional con rendimientos de $7.8 \mathrm{t}$ ha1, producción que ha venido disminuyendo desde el año 2011 (Agronet, 2016).

La disminución en los rendimientos se debe a una serie de problemas que involucran el manejo agronómico (Suarez y Mederos, 2011), manejo de las propiedades de los suelos (Serrano et al., 2017), la transferencia de tecnología, la limitada capacitación, insuficiente apoyo económico y desconocimiento de las zonas y variedades con mayor aptitud productiva para este cultivo, entre otros (Rodríguez, 2013). El rendimiento de la yuca se determina por la tasa de producción de biomasa seca y por su eficiencia en acumular fotoasimilados en las raíces tuberosas, por lo tanto, la capacidad fotosintética de la planta es de gran importancia en la productividad de esta (Ramanujan, 1990). Durante los primeros meses del cultivo, la formación de hojas tiene prioridad sobre el crecimiento de raíces de almacenamiento; después la planta continúa formando hojas a la vez que almacena almidón en las raíces (Mejía et al., 2002). El porcentaje de materia seca y el contenido de almidón, comúnmente considerados factores de calidad, varían mucho entre las distintas variedades de yuca. Además, se ha observado que los factores descritos están muy ligados principalmente con el contenido de potasio del suelo, con la edad del cultivo, el clima, principalmente la lluvia y contenido de humedad del suelo (Marín et al., 2008).

Dentro de la especie M. esculenta se ha identificado una amplia variabilidad genética, que incluyen rasgos de naturaleza agronómica y morfológica, resistencia a las principales plagas y enfermedades y condiciones de calidad, entre otros. Estos aspectos dificultan el estudio del cultivo, debido a que debe ser evaluado no solo por su comportamiento varietal sino también por su respuesta al ambiente sobre el cual es producido. Para esta investigación se ha planteado como objetivo la evaluación de algunos parámetros fisiológicos del cultivo de Yuca en la zona de bosque seco tropical en el departamento de Sucre.

\section{METODOLOGÍA}

Esta investigación se llevó a cabo en el departamento de Sucre, en la zona de bosque seco tropical, bajo condiciones ambientales que se distribuyen entre los 0-1000 msnm de 
altitud, presenta temperaturas superiores a los $24^{\circ} \mathrm{C}$ (piso térmico cálido) y precipitaciones entre los 700 y 2000 mm anuales, con uno o dos periodos marcados de sequía al año (Aguilera, 2012). Los ensayos se realizaron en

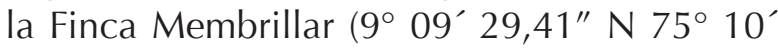
09,66" O), Santa Fe (9 $9^{\circ} 20^{\prime} 38,15^{\prime \prime} N 75^{\circ} 18^{\prime}$ $\left.40,06^{\prime \prime} \mathrm{O}\right)$ y Arenal (9० $18^{\prime} 44,35^{\prime \prime}$ N. $75^{\circ} 32^{\prime}$ $48,39^{\prime \prime}$ O) en los municipios Corozal, Morroa y San Antonio de Palmito respectivamente.

Las características edáficas de las fincas en estudio se muestran en la tabla 1. Las condiciones ambientales promedio en cada finca, fueron medidas con estaciones meteorológicas portátiles, evidenciando que en el periodo del ensayo, comprendido entre octubre del 2015 y octubre del 2016, se tuvieron temperaturas máximas que oscilan entre los 33,4 y $38{ }^{\circ} \mathrm{C}$, temperatura mínimas entre 21,4 y $23{ }^{\circ} \mathrm{C}$ y una precipitación total de $473,9 \mathrm{~mm}$, inferior a la media característica de la zona, por efectos del fenómeno del niño presente en ese año de trabajo.

La menor cantidad precipitaciones se presentaron en el periodo comprendido entre los meses de diciembre de 2015 y marzo de 2016 (época de sequía), en los cuales solo se aportó solo el $5 \%$ del total de precipitaciones en el ciclo del cultivo (25 mm). La mayor disponibilidad hídrica para el cultivo estuvo entre octubre y noviembre de 2015, y entre agosto y octubre de 2016, en los cuales ocurrió el $86 \%$ de las precipitaciones presentes en el ciclo del cultivo.

Se evaluaron tres variedades de yuca: MCOL 2066 (Chirosa), MCOL 2215 (Venezolana) y M-Tai 8 (M-Tai), en el periodo comprendido entre de octubre de 2015 y octubre de 2016. Las labores de preparación del suelo fueron efectuadas bajo el sistema convencional, realizando un pase de arado de disco, un pase de rastra de disco y surcado. La siembra se efectuó de forma manual a $1 \mathrm{~m}$ entre hileras y $1 \mathrm{~m}$ entre plantas, para una densidad de población de 10000 plantas ha ${ }^{-1}$ La investigación fue realizada bajo un diseño experimental en bloques completos al azar con tres repeticiones, donde cada una de las localidades se tomó con un bloque o repetición para la caracterización de la respuesta del bosque seco tropical del departamento de Sucre. En cada una de las localidades se establecieron los cultivos con un tamaño de unidad experimental de $25 \times 24 \mathrm{~m}$, para un total de 600 plantas por unidad experimental.

Se realizaron cuatro muestreos en el ciclo del cultivo, uno en la primera fase de crecimiento y desarrollo (90 DDS) y tres muestreos en época de cosecha (270, 315, y 360 DDS). Para las siguientes variables: Variables morfológicas: fueron medidas en tres plantas en plena competencia de la parcela útil por unidad experimental de la siguiente manera: la Altura de plantas (AP: la longitud desde la superficie de suelo hasta la última hoja desplegada por la planta); diámetro del tallo (DT: diámetro del tallo a $5 \mathrm{~cm}$ de la superficie del suelo); número de hojas $(\mathrm{NH})$; diámetro de raíces tuberosas (DR: diámetro promedio de tres raíces tuberosas por planta muestreada).

Intercambio gaseoso: se cuantifico con el sistema de medición infrarrojo de gases, IRGA, entre las 8 am y 11 am, en una hoja joven completamente desplegada del tercio medio de tres plantas en plena competencia en la parcela útil de cada unidad experimental, manteniendo constante la intensidad de la luz en 1200 $\mu \mathrm{mol} \mathrm{m} \mathrm{m}^{-2} \mathrm{~s}^{-1}$ y la concentración de $\mathrm{CO}^{2}$ en 400 $\mu \mathrm{mol} \mathrm{mol}^{-1}$, realizando las lecturas de las variables $P N$ : Fotosíntesis neta de la hoja, $G S$ : Conductancia estomática y $E$ : transpiración.

Acumulación, distribución de biomasa e índice de cosecha: Para las variables de acumulación y distribución de biomasa se realizaron muestreos destructivos de tres plantas en plena competencia de la parcela útil por unidad experimenta, y se midió el peso 
fresco de cada uno de los órganos de las tres plantas (raíces tuberosas, tallos y hojas), de las cuales se tomaron muestra de 150 a 200 g de cada uno de los órganos de las plantas, llevadas a secado en hornos durante 72 horas, a una temperatura constante de $70{ }^{\circ} \mathrm{C}$.

El índice de cosecha fue calculado con la relación entre la biomasa del órgano de interés (raíces tuberosas) y el total de biomasa acumulada por la planta. Todos los datos fueron analizados en el programa estadístico SAS 9.1, bajo los lineamientos del diseño experimental mencionado anteriormente, realizando los análisis de varianzas y las comparaciones de medias para cada una de las variables, con la prueba de Tukey con una 5\% de probabilidad.

\section{RESULTADOS Y DISCUSIÓN}

Se sabe qué el crecimiento y desarrollo de las plantas depende de la división, expansión y diferenciación celular, todos estos proceso son afectados por el déficit hídrico, debido a la perdida de turgencia (Tezara et al., 2002), las condiciones hídricas en la zona de estudio durante el ciclo del cultivo fueron muy inferiores a los valores medios características de la zona de bosque seco tropical del departamento de Sucre, y por lo tanto inferiores las reportadas en estudios realizados por Pastrana et al. (2015), Quien expresa que para obtener los mejores rendimientos y desarrollo de los cultivos de yuca en el bosque seco del departamento se deben tener alrededor de $1247 \mathrm{~mm}$ de agua disponibles en el ciclo del cultivo, por lo tanto las plantas del ensayo estuvieron sometidas a condiciones de estrés hídrico.

Variables morfológicas. En las variables morfológicas evaluadas a los 90 DDS (Tabla 1) se observa un efecto significativo de la variedad de yuca sobre la AP y $\mathrm{NH}$, mientras que en las variables DT y DR tuberosas no se muestran diferencias estadísticas. La mayor altura de planta fue observada en la variedad M-Tai, con un promedio de $85,83 \mathrm{~cm}$, superando en $24,87 \mathrm{~cm}$ a las demás variedades, que mostraron menor porte a dicha edad, siendo iguales estadísticamente entre ellas, con una media de 60,96 cm de altura. El NH, fue mayor en las variedades Venezolana y M-Tai, con 92 hojas por planta, por su parte Chirosa expuso en promedio 39 hojas menos que las demás. Además, no se observan diferencias estadísticas para la variable morfológica de diámetro de las raíces tuberosas, mostrando un comportamiento similar entre las diferentes variedades evaluadas, con diámetros medio de 17,83 mm. Las estructuras morfológicas de la yuca dependen de la expresión de los genotipos como respuesta a las condiciones ambientales, en las cuales pueden variar diámetros y longitudes de tallos y raíces, número y alturas de ramificaciones Gabriel et al. (2014). Conforme con los resultados, a los 90 DDS, las variedades de uso industrial M-Tai, y la de consumo humano (Venezolana) poseen mayor número de hojas en etapas juveniles, en comparación con la Chirosa, resaltando un mayor desarrollo de dosel en M-Tai (mayor altura de planta y número de hojas), teniendo en cuenta que la altura de la planta es un descriptor morfológico fundamental relacionado con la expresión del carácter genotípico (Fukuda y Guevara, 1998) y que la nutrición mineral estimula la formación de los sistemas radiculares, el crecimiento, los contenidos de carbohidratos y proteínas en las plantas de yuca, mejorando el aprovechamiento de agua y viceversa (Coqueiro, 2013). Una de las razones por la que la variedad M-Tai alcanzo un mayor desarrollo del dosel puede ser el uso más eficiente de los minerales y mayor facilidad para realizar labores de manejo agronómico, que puede verse reflejado en mejorar la productividad del cultivo (Cenoz et al., 2016). Los resultados de las variables morfológicas a los 270 DDS (Tabla 2) muestran un efecto significativo de la variedad de yuca sobre la 
altura de planta, mientras que las variables número de hojas, diámetro de tallo y diámetro de raíces tuberosas no muestran diferencias estadísticas. Las mayores alturas de planta con valores de 214,4 cm fueron observadas en las variedades Chirosa y M-Tai, siendo iguales estadísticamente entre ellas. En el mismo sentido la variedad M-Tai presentó altura estadísticamente similar a la variedad Venezolana, que mostró menor porte a dicha edad con una altura de $185 \mathrm{~cm}$ en promedio. La variable número de hojas y raíces tuberosas no presentaron diferencia significativa, mostrando un comportamiento similar entre las diferentes variedades evaluadas encontrando un número de hoja promedio de 180,5 y diámetro medio de 44,97 mm. Según los resultados las tres variedades de yuca evaluadas muestran diferencias sólo en la variable altura de planta a los 270 DDS, presentando mayor desarrollo de esta variable el clon Chirosa en comparación al clon Venezolana, indicando una mejor expresión genética de este bajo las condiciones de bosque seco tropical para esta etapa del cultivo (Beovides et al., 2013). Las alturas presentadas por las variedades Venezolana y M-Tai son similares a los valores medios entre 134 y $187 \mathrm{~cm}$ presentados en esta misma etapa, en una plantación de yuca tratada con micorrizas arbusculares bajo condiciones similares (João et al., 2016).

Los resultados de las variables morfológicas a los 315 DDS (Tabla 2) muestran un efecto significativo de la variedad de yuca sobre la altura de planta y número de hojas, mientras que en las variables diámetro de tallo y diámetro de raíces tuberosas no se muestran diferencias estadísticas. La mayor altura de planta fue observada en la variedad Chirosa, con valores promedios de $261 \mathrm{~cm}$, la cual supera en $51,5 \mathrm{~cm}$ a las demás variedades, que mostraron menor porte a dicha edad, siendo iguales estadísticamente entre ellas, con una media de 209,53 cm de altura. Además, el número de hojas, fue mayor en las variedades Venezolana y Chirosa, con 268 hojas por planta, por su parte la variedad M-Tai expuso en promedio de 102 hojas menos que las demás. Además, la variable diámetro de raíces tuberosas no muestran diferencia significativa, con un comportamiento similar entre las diferentes variedades evaluadas encontrando diámetros medio de 53,88 mm. A los 315 DDS, el mayor número de hojas en etapas adultas las presentan las variedades de consumo humano (Venezolana y Chirosa), en comparación con la M-Tai; además el clon Chirosa presenta mayor altura de planta que los demás clones estudiados, podría deberse a su expresión genotípica (Beovides et al., 2013) al uso eficiente de los minerales (Coqueiro, 2013).

Tabla 1. Características fisicoquímicas de los suelos de bosque seco tropicl de Sucre, Colombia.

\begin{tabular}{|c|c|c|}
\hline MUNICIPIO & PARÁMETROS & VALOR \\
\hline \multirow[t]{2}{*}{ Corozal } & \multirow{2}{*}{$\begin{array}{l}\text { Químicos } \\
\text { PH } \\
\text { M.O (\%) } \\
\text { P (ppm) } \\
\left.\text { K (cmol } \mathrm{kg}^{-1}\right) \\
\mathrm{Ca}\left(\mathrm{cmol}^{+} \mathrm{kg}^{-1}\right) \\
\mathrm{Na}\left(\mathrm{cmol}^{+} \mathrm{kg}^{-1}\right) \\
\mathrm{CIC}\left(\mathrm{cmol}^{+} \mathrm{kg}^{-1}\right) \\
\text { Físicos } \\
\text { Textura } \\
\text { Da }\left(\mathrm{g} \mathrm{cm}^{-3}\right)\end{array}$} & $\begin{array}{l}6,01 \\
1,61 \\
7,73 \\
0,16 \\
9,4 \\
2,2 \\
19,0\end{array}$ \\
\hline & & $\begin{array}{l}\text { Franco arcilloso } \\
1,68\end{array}$ \\
\hline \multirow[t]{2}{*}{ Morroa } & \multirow{2}{*}{$\begin{array}{l}\text { Químicos } \\
\text { PH } \\
\text { M.O (\%) } \\
\text { P (ppm) } \\
\text { K }\left(\mathrm{cmol}^{+} \mathrm{kg}^{-1}\right) \\
\mathrm{Ca}\left(\mathrm{cmol}^{+} \mathrm{kg}^{-1}\right) \\
\mathrm{Na}\left(\mathrm{cmol}^{+} \mathrm{kg}^{-1}\right) \\
\mathrm{CIC}\left(\mathrm{cmol}^{+} \mathrm{kg}^{-1}\right) \\
\text { Fí́cicos } \\
\text { Textura } \\
\text { Da }\left(\mathrm{g} \mathrm{cm}^{-3}\right)\end{array}$} & $\begin{array}{l}5,82 \\
2,23 \\
20,5 \\
0,22 \\
12,0 \\
2,1 \\
18,5\end{array}$ \\
\hline & & $\begin{array}{l}\text { Franco arcilloso } \\
1,54\end{array}$ \\
\hline \multirow[t]{2}{*}{$\begin{array}{l}\text { San Antonio de } \\
\text { Palmito }\end{array}$} & \multirow{2}{*}{$\begin{array}{l}\text { Químicos } \\
\text { PH } \\
\text { M.O (\%) } \\
\text { P (ppm) } \\
\text { K }\left(\mathrm{cmol}^{+} \mathrm{kg}^{-1}\right) \\
\mathrm{Ca}\left(\mathrm{cmol}^{+} \mathrm{kg}^{-1}\right) \\
\mathrm{Na}\left(\mathrm{cmol}^{+} \mathrm{kg}^{-1}\right) \\
\mathrm{CIC}\left(\mathrm{cmol}^{+} \mathrm{kg}^{-1}\right) \\
\text { Físicos } \\
\text { Textura } \\
\text { Da }\left(\mathrm{g} \mathrm{cm}^{-3}\right)\end{array}$} & $\begin{array}{l}6,04 \\
1,63 \\
15,03 \\
0,12 \\
9,4 \\
2,0 \\
16,0\end{array}$ \\
\hline & & $\underset{1,63}{\text { Franco arcilloso }}$ \\
\hline
\end{tabular}

En las variables morfológicas evaluadas a los 360 días después de la siembra (Tabla 2) se observa un efecto significativo de la variedad de yuca sobre el número de hojas, mientras que en las variables altura de planta, diámetro de tallo y diámetro de raíces tuberosas 
Tabla 2. Comparaciones de medias para las variables de crecimiento del cultivo de Manihot esculenta Crantz en el Bosque seco Tropical del departamento de Sucre - Colombia, 2016.

\begin{tabular}{|c|c|c|c|c|}
\hline \multicolumn{4}{|c|}{$\begin{array}{l}\text { DDS VARIEDAD VENEZOLANA M - TAI } \\
\text { VARIABLE }\end{array}$} & CHIROSA \\
\hline 90 & $\begin{array}{l}\mathrm{NH} \\
\text { AP } \\
\text { DT } \\
\text { DR }\end{array}$ & $\begin{array}{r}85,11 \mathrm{a} \\
59,00 \mathrm{~b} \\
1,39 \mathrm{a} \\
16,20 \mathrm{a}\end{array}$ & $\begin{array}{r}102,50 \mathrm{a} \\
85,83 \mathrm{a} \\
1,16 \mathrm{a} \\
27,30 \mathrm{a}\end{array}$ & $\begin{array}{r}53,00 \mathrm{~b} \\
64,00 \mathrm{~b} \\
1,23 \mathrm{a} \\
16,98 \mathrm{a}\end{array}$ \\
\hline 270 & $\begin{array}{l}\mathrm{NH} \\
\text { AP } \\
\text { DT } \\
\text { DR }\end{array}$ & $\begin{array}{r}130,50 \mathrm{a} \\
185,17 \mathrm{~b} \\
22,24 \mathrm{a} \\
42,45 \mathrm{a}\end{array}$ & $\begin{array}{c}165,00 \mathrm{a} \\
196,33 \mathrm{ab} \\
21,18 \mathrm{a} \\
43,57 \mathrm{a}\end{array}$ & $\begin{array}{r}246,00 \mathrm{a} \\
232,44 \mathrm{a} \\
23,80 \mathrm{a} \\
48,90 \mathrm{a}\end{array}$ \\
\hline 315 & $\begin{array}{l}\mathrm{NH} \\
\mathrm{AP} \\
\mathrm{DT} \\
\mathrm{DR}\end{array}$ & $\begin{array}{r}245,5 \mathrm{a} \\
216,83 \mathrm{~b} \\
23,20 \mathrm{a} \\
59,12 \mathrm{a}\end{array}$ & $\begin{array}{r}166,00 \mathrm{~b} \\
202,22 \mathrm{~b} \\
20,47 \mathrm{a} \\
51,93 \mathrm{a}\end{array}$ & $\begin{array}{r}290,67 \mathrm{a} \\
261,00 \mathrm{a} \\
24,60 \mathrm{a} \\
50,58 \mathrm{a}\end{array}$ \\
\hline 360 & $\begin{array}{l}\mathrm{NH} \\
\text { AP } \\
\text { DT } \\
\text { DR }\end{array}$ & $\begin{array}{r}459,50 \mathrm{a} \\
230,17 \mathrm{a} \\
24,28 \mathrm{a} \\
57,85 \mathrm{a}\end{array}$ & $\begin{array}{r}200,33 \mathrm{~b} \\
220,67 \mathrm{a} \\
22,76 \mathrm{a} \\
61,62 \mathrm{a}\end{array}$ & $\begin{array}{r}289,00 \mathrm{a} \\
259,00 \mathrm{a} \\
25,31 \mathrm{a} \\
57,19 \mathrm{a}\end{array}$ \\
\hline
\end{tabular}

Medias con igual letra en columna no mostraron diferencia significativa conforme la prueba de Tukey $(\boldsymbol{\alpha}=0.05)$. Las comparaciones son realizadas horizontalmente. Número de hojas $(\mathrm{NH})$, altura de plantas: AP $(\mathrm{cm})$, diámetro del tallo: DT $(\mathrm{cm})$, diámetro de raíces tuberosas: DR $(\mathrm{mm})$.

no se muestran diferencias estadísticas. El mayor número de hojas, fue mayor en la variedades Venezolana y Chirosa con 374,25 hojas por planta, por su parte la variedad M-Tai expuso en promedio de 173,92 hojas menos que las demás. La variable altura de planta no muestra diferencia significativa, mostrando un comportamiento similar entre las variedades evaluadas encontrando altura media de plantas alrededor de los 236,61 cm.

Adicionalmente en la tabla 2, no se observan diferencias estadísticas para la variable morfológica de las raíces tuberosas, teniendo un comportamiento similar entre las diferentes variedades evaluadas, encontrando diámetros medio de 52,89 mm. Después de un año establecido el cultivo los resultados muestran que la única variable morfológica que muestra diferencia entre variedades es el número de hojas, en donde los clones de consumo humano (Venezolana y Chirosa) presentan mayores valores, en comparación con la M-Tai; Se sabe que la respuesta de la yuca en cuanto al número, peso y calidad de sus raíces y hojas o la altura de la planta, además de su componente genético, está muy vinculada a indicadores medioambientales como las Iluvias, la temperatura o la luz (Folgueras et al., 2010). A partir los resultados se puede afirmar que para esta etapa del cultivo las variedades han equilibrado las medidas de las demás las variables morfológicas estudiadas. Las alturas presentadas por las variedades estudiadas son similares a los valores medios de $219 \mathrm{~cm}$ en esta misma etapa en los resultados obtenidos por João et al. (2016) y Beovides et al. (2014) bajo condiciones similares tanto varietales y ambientales.

Intercambio gaseoso. Después de realizado el análisis de varianza para las variables evaluadas de intercambio (Gs: conductancia estomática, $P N$ : fotosíntesis neta, $E$ : transpiración), no se presentaron diferencias significativas entre las variedades, en cada uno de los muestreos realizados (Tabla 3), observando un comportamiento similar entre las variedades con medias de $130 \mathrm{mmol} \mathrm{H}_{2} \mathrm{O} \mathrm{m}^{-2} \mathrm{~s}^{-1}, 17,87$ umoles $\mathrm{CO}_{2} \mathrm{~m}^{-2} \mathrm{~s}^{-1}$ y $1,99 \mathrm{mmol} \mathrm{H}_{2} \mathrm{O} \mathrm{m}^{-2} \mathrm{~s}^{-1}$ para $G S, P N$ y $E$ respectivamente, para el primer muestreo 90 DDS. A los 270, 315 y 369 DDS se observó una tendencia al aumento de la conductancia foliar y la transpiración mientras que la fotosíntesis se mantiene relativamente estable, teniendo valores de $G S$ que oscilan entre 480 y $565 \mathrm{mmol} \mathrm{H}_{2} \mathrm{O} \mathrm{m}^{-2} \mathrm{~s}^{-1} \mathrm{y}$ de $P N$ entre los 12 y 25 umoles $\mathrm{CO}_{2} \mathrm{~m}^{-2} \mathrm{~s}^{-1}$.

Observando el comportamiento del intercambio gaseoso de las tres variedades, se puede afirmar que todas tienen la misma capacidad de responder a las condiciones ambientales, para estas variables, siendo estadísticamente iguales, con valores de conductancia estomática entre los rangos reportados por Da Silva et al. (2010) entre 55- $359 \mathrm{mmol} \mathrm{H}_{2} \mathrm{O} \mathrm{m}^{-2} \mathrm{~s}^{-1}$ para plantas de yuca en condiciones de humedad optimas y con estrés hídrico respectivamente, explicando los valores bajos del ensayo en respuesta a la disponibilidad hídrica presente en la zona de estudio para el primer muestreo. 
Para fotosíntesis neta $(P N)$ los datos resultantes estuvieron entre $12-25 \mu \mathrm{mol} \mathrm{CO}_{2} \mathrm{~m}^{-2} \mathrm{~s}^{-1}$, que resultan menores al compararlos con los obtenidos por Holguin et al. (1984) que están alrededor de los 26 - $32 \mu \mathrm{mol} \mathrm{CO}_{2} \mathrm{~m}^{-2} \mathrm{~s}^{-1} \mathrm{en}$ su tratamiento testigo, en plantas sanas y en buenas condiciones hídricas. Mejía (2002) indica que las hojas de la yuca tienen una

Tabla 3. Comparaciones de medias para las variables de crecimiento del cultivo de Manihot esculenta Crantz en el Bosque seco Tropical del departamento de Sucre - Colombia, 2016.

\section{DDS VARIEDAD VENEZOLANA M - TAI CHIROSA} VARIABLE

\begin{tabular}{rrrrr}
\hline \multirow{4}{*}{90} & $G S$ & $133,67 \mathrm{a}$ & $111,75 \mathrm{a}$ & $145,00 \mathrm{a}$ \\
& $E$ & $2,14 \mathrm{a}$ & $1,84 \mathrm{a}$ & $1,87 \mathrm{a}$ \\
& $P N$ & $21,59 \mathrm{a}$ & $12,20 \mathrm{a}$ & $24,43 \mathrm{a}$ \\
270 & $G S$ & $259,00 \mathrm{a}$ & $148,67 \mathrm{a}$ & $188,89 \mathrm{a}$ \\
& $E$ & $2,41 \mathrm{a}$ & $1,51 \mathrm{a}$ & $1,84 \mathrm{a}$ \\
& $P N$ & $24,08 \mathrm{a}$ & $13,61 \mathrm{a}$ & $15,38 \mathrm{a}$ \\
315 & $G S$ & $395,67 \mathrm{a}$ & $561,89 \mathrm{a}$ & $462,89 \mathrm{a}$ \\
& $E$ & $4,59 \mathrm{a}$ & $5,16 \mathrm{a}$ & $4,93 \mathrm{a}$ \\
& $P N$ & $32,72 \mathrm{a}$ & $15,82 \mathrm{a}$ & $15,67 \mathrm{a}$ \\
360 & $G S$ & $3,71 \mathrm{a}$ & $387,89 \mathrm{a}$ & $281,78 \mathrm{a}$ \\
& $E$ & $13,90 \mathrm{a}$ & $16,66 \mathrm{a}$ & $3,49 \mathrm{a}$ \\
& $P N$ & $13,62 \mathrm{a}$ \\
\hline
\end{tabular}

Medias con igual letra en columna no mostraron diferencia significativa conforme la prueba de Tukey $(\boldsymbol{\alpha}=0.05)$. Las comparaciones se realizan de forma horizontal. Gs: conductancia estomática $\left(\mathrm{mmol} \mathrm{H}_{2} \mathrm{O} \mathrm{m}^{-2} \mathrm{~s}^{-1}\right), P N$ : fotosíntesis neta $\left(\mu\right.$ moles $\left.\mathrm{CO}_{2} \mathrm{~m}^{-2} \mathrm{~s}^{-1}\right),+$ : transpiración $\left(\mathrm{mmol} \mathrm{H}_{2} \mathrm{O} \mathrm{m}^{-2} \mathrm{~s}^{-1}\right)$.

El-Sharkawy (2012) y León et al. (2014) indicaron en ambientes con períodos de precipitación estacional y con valores mayores a $900 \mathrm{~mm}$ año, tasa de fotosíntesis entre 25 y $31 \mu \mathrm{mol} \mathrm{CO}_{2} \mathrm{~m}^{-2} \mathrm{~s}^{-1}$ en cultivares de yuca; mientras que en ambiente semiárido y con lluvias menores a $600 \mathrm{~mm}$ año, las tasas de fotosíntesis variaron desde 7 hasta $20 \mu \mathrm{mol} \mathrm{CO}_{2}$ $\mathrm{m}^{-2} \mathrm{~s}^{-1}$ entre los clones evaluados. Estos autores afirman que la capacidad fotosintética de la yuca puede estar relacionada con hábitat de origen de los cultivares y con el cierre parcial de los estomas. Se puede decir entonces que una de las razones de los valores bajos resultantes de este ensayo en la variable fotosíntesis puede ser las bajas condiciones de humedad del suelo.

Las plantas de yuca disminuyeron la conductancia estomática, y por ende la pérdida de agua por transpiración y permitieron el intercambio de $\mathrm{CO}_{2}$ para la fotosíntesis bajo las condiciones de humedad desfavorables, hídricamente hablando (León et al., 2017).

cumulación, distribución de biomasa e índice de cosecha. En la tabla 4 se observan las comparaciones de media para la variable acumulación de biomasa por órganos de las plantas. A los 90 DDS no se observan diferencias estadísticas para las medias de acumulación de biomasa en hojas (PSH), con valores entre 72,48 y 100,66 gr de materia Adicionalmente se presentan diferencias estadísticas para las variables peso seco de tallos y peso seco de raíces tuberosas, en los cuales las plantas de mayor acumulación de biomasa en tallos fueron las de la variedad M-Tai, con valores alrededor de 85,74 gr, mientras que las variedades Venezolana $y$ Chirosa, presentaron una menor acumulación de biomasa en este órgano, con una media de 38,09 gr por planta. Para la variable Peso seco de raíces tuberosas, a los 90 días después de la siembra, se obtuvo en el clon M-Tai una media de 198,51 gr de materia seca, pudiendo ser solo igualada estadísticamente por la variedad Venezolana, pero este a su vez no presenta diferencias estadísticas con la variedad Chirosa.

Tabla 4. Comparaciones de medias para las variables de distribución y acumulación biomasa seca por órgano en plantas de yuca (Manihot esculenta Crantz) en el Bosque seco Tropical del departamento de Sucre - Colombia, 2016.

\begin{tabular}{|c|c|c|c|c|}
\hline DD & $\begin{array}{l}\text { IED } \\
\text { IAI }\end{array}$ & EZOL & $1-\mathrm{T}$ & CHIROS \\
\hline 90 & $\begin{array}{l}\text { PSH } \\
\text { PST } \\
\text { PSR }\end{array}$ & $\begin{array}{r}100,66 \mathrm{a} \\
37,25 \mathrm{~b} \\
8,27 \mathrm{ab}\end{array}$ & $\begin{array}{r}73,41 \mathrm{a} \\
85,74 \mathrm{a} \\
198,51 \mathrm{a}\end{array}$ & $\begin{array}{l}72,48 \mathrm{a} \\
32,70 \mathrm{~b} \\
34,71 \mathrm{~b}\end{array}$ \\
\hline 270 & $\begin{array}{l}\text { PSH } \\
\text { PST } \\
\text { PSR } \\
\text { IC }\end{array}$ & $\begin{array}{r}186,91 \mathrm{a} \\
329,65 \mathrm{a} \\
710,67 \mathrm{a} \\
0,57 \mathrm{a}\end{array}$ & $\begin{array}{r}186.91 \mathrm{a} \\
329.65 \mathrm{a} \\
710.67 \mathrm{a} \\
0,57 \mathrm{a}\end{array}$ & $\begin{array}{r}228,56 \mathrm{a} \\
533,64 \mathrm{a} \\
928,65 \mathrm{a} \\
0,55 \mathrm{a}\end{array}$ \\
\hline 315 & $\begin{array}{c}\text { PSH } \\
\text { PST } \\
\text { PSR } \\
\text { IC }\end{array}$ & $\begin{array}{r}141,45 \mathrm{a} \\
604,42 \mathrm{a} \\
902,48 \mathrm{a} \\
0,56 \mathrm{a}\end{array}$ & $\begin{array}{r}162,78 \mathrm{a} \\
922,93 \mathrm{a} \\
1319,92 \mathrm{a} \\
0,54 \mathrm{a}\end{array}$ & $\begin{array}{r}176,50 \mathrm{a} \\
859,65 \mathrm{a} \\
1102,19 \mathrm{a} \\
0,51 \mathrm{a}\end{array}$ \\
\hline 360 & $\begin{array}{c}\text { PSH } \\
\text { PST } \\
\text { PSR } \\
\text { IC }\end{array}$ & $\begin{array}{r}198,98 \mathrm{a} \\
843,75 \mathrm{a} \\
1365,91 \mathrm{a} \\
0,56 \mathrm{a}\end{array}$ & $\begin{array}{r}155,55 \mathrm{a} \\
743,28 \mathrm{a} \\
1481,35 \mathrm{a} \\
0,54 \mathrm{a}\end{array}$ & $\begin{array}{r}152,11 a \\
819,20 a \\
1345,72 a \\
0,51 a\end{array}$ \\
\hline
\end{tabular}

Medias con igual letra en columna no mostraron diferencia significativa conforme la prueba de Tukey $(\alpha=0.05)$. PSH: Peso seco de raíces, PST: peso seco de tallo, PSR: peso seco de raíces tuberosas, IC: índice de cosecha. 
capacidad fotosintética relativamente seca. de Entre los 270, 315, 360 DDS, no se observan diferencias significativas para ninguna de las variables evaluadas pero se evidencia una tendencia al aumento de la biomasa de raíces y tallos, mientras que en las hojas se observa una tendencia inversa, disminuyendo peso de las

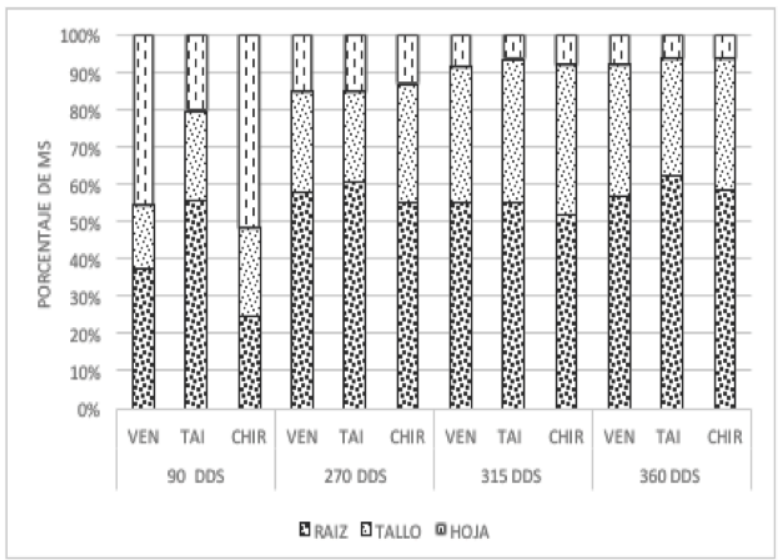

Figura 1. Distribución porcentual de acumulación de materia seca por órganos de la planta de yuca (Manihot esculenta Crantz), a los 90, 270, 315 y 360 DDS en Bosque seco Tropical del departamento de Sucre - Colombia. 2016. .

hojas al transcurrir el tiempo (Tabla 4 y Figura 1). Para las variables de acumulación de biomasa en hojas, no se observaron diferencias estadísticas, acorde con los estudios realizados por Coqueiro (2013) quien demostró que este comportamiento es explicado de acuerdo a las características propias de los genotipos evaluados. Por otra parte, Sagrilo et al. (2002) reportan que la producción de fitomasa depende de la respuesta genotipo-ambiente, de tal forma que en las condiciones de este ensayo se favoreció la expresión de genes en las plantas de M-Tai mostrando mayor acumulación de biomasa en tallos y raíces, y en Venezolana en la acumulación de materia seca en raíces, siendo igual estadísticamente a la M-Tai, para esta esta variable.

Estos resultados muestran claramente cómo se diferencia el crecimiento y desarrollo de las plantas de yuca como respuesta al fenotipo mostrado por cada una de las variedades evaluados, y que la acumulación de materia seca en las raíces tuberosas depende de la capacidad de trastocar los asimilados al órgano de interés y esto a su vez de la influencia por la capacidad del sumidero de las partes de reserva (Alves, 2002); notándose una similar respuesta de las variedades del estudio en la época de cosecha, aunque a los 90 DDS la variedades M-Tai y Venezolana, de uso industrial y de consumo humano respectivamente, presentan una mejor distribución de los asimilados a los órganos de interés estando al rededor del 35 y 55\% del total de biomasa acumulada; en comparación con la Chirosa con los dos usos mencionados, en los cuales la acumulación de biomasa en el órgano de interés está alrededor de $20 \%$ del total, pero siendo mayor de lo reportado por Alves (2002), quien expresa que de los 60 a 75 días las raíces tuberosas representan del 10 al $15 \%$ de la metería seca total de la planta, y en esta etapa inicia una acumulación rápida de asimilados a las raíces tuberosas hasta llegar a representar proporción del 50 al $60 \%$ de la materia seca total, que fue lo que observó en los últimos estadios del ciclo del cultivo de este ensayo.

A pesar de las diferencias observadas en la acumulación de fitomasa en el órgano de interés y el efecto las condiciones edafoclimaticas se pueden considerar como buenos los índices de cosecha obtenidos, para la fecha de muestreo, siendo acordes a los reportados por Coqueiro (2013), quien considera un buen índice de cosecha del 50\% para estadios finales.

Respecto a la distribución de biomasa por planta (Figura 1), se observa una mejor respuesta de los clones M-Tai y Venezolana, a los 90 DDS, en los cuales se presentó una mejor distribución de los asimilados a los órganos de interés estando al rededor del 35 y $55 \%$ del total de biomasa acumulada; en comparación con la variedad Chirosa, en el cual la acumulación de biomasa en el órgano de interés está alrededor de $20 \%$ del total de biomasa acumulado. La distribución de biomasa por planta a los 
270, 315 y 360 DDS (Figura 1), muestra que los clones evaluados M-Tai, Venezolana y Chirosa presentaron similitud en la distribución de los asimilados a los órganos de interés estando alrededor del $55 \%$ del total de biomasa acumulada (Figura 1).

\section{CONCLUSIONES}

El crecimiento y desarrollo de las variedades de yuca evaluadas bajo la oferta ambiental del Bosque Seco Tropical del departamento de Sucre varía; los aspectos morfológicos del crecimiento vegetal, acumulación de biomasa y capacidad fotosintéticas propias de cada genotipo, permite inferir que las plantas de M-Tai tienen un crecimiento y desarrollo más acelerado que las otras dos variedades (Chirosa y Venezolana), evidenciándose en una mayor acumulación de materia seca en los primeros estadios de su crecimiento. Estas tres variedades de yuca tuvieron la misma capacidad de intercambio gaseoso y de distribución de biomasa al órgano de interés.

\section{REFERENCIAS}

Agronet. 2016. Estadística del cultivo de la yuca. Evaluaciones agropecuarias municipales. Oficina de Planeación y Prospectiva MADR. http://www. agronet.gov.co/estadistica/Paginas/ default.aspx (consultado 21 Nov. 2018).

Aguilera, M. 2012. La yuca en el Caribe colombiano: De cultivo ancestral a agroindustrial. Banco de la república-economía regional. p.158.

Alves, A. 2002. Cassava botany and physiology. In: R.J. Hillocks. Editors, Cassava: Biology, Production and Utilization.1nd ed. CABI Publishing, GBR, p. 67-89.

Beovides, Y., Milián, M. D., Rodríguez, D., Gálvez, L., Rodríguez, K., Fernández, M. I. y Oliva, M. 2013. Cultivares cubanos de yuca (Manihot esculenta Crantz) con rendimiento y potencial genético para la agroindustria.
Centro Agrícola, 40(3), 71-78. Beovides, G., Milián, J., Coto, O., Rayas, A., Basail, M., Santos, A. y Ruiz, E. 2014. Caracterización morfológica y agronómica de cultivares cubanos de yuca (Manihot esculenta Crantz). Cultivos Tropicales, 35(2), 43-50..

Cenoz, P., López, A. y Burgos, A. 2016. Efecto de los macro nutrientes en el desarrollo y rendimiento de Mandioca (Manihot esculenta, Crantz). Agrotecnia, (7), 9-12.

Coqueiro, G. 2013. Avaliação de variedades de mandioca no nordeste do Estado do Pará. Teses doutorado Universidade de Estadual Paulista "Júlio De Mesquita Filho, Brasil.

Da Silva, J., Da Silva, C., Filho, M. y De Jesus, S. 2010. Desempenho fisiológico de genótipos de mandioca submetidos a estresse hídrico. Teses Doutorado da em Ciências Agrárias. UFRB.

El-Sharkawy, M. 2012. Stress-Tolerant Cassava: The Role of Integrative EcophysiologyBreeding Research in Crop Improvement. Open Journal of Soil science 2 (2): 162-186

FAOSTAT. 2016. Agriculture Organization. Roma, Italia. http://faostat.external.fao. org/. (Consultado 21 de Nov. 2018).

FAO. 2008. Yuca para la seguridad alimentaria $y$ energética. Food and Agriculture Organization of the United Nations. Yuca para la seguridad alimentaria y energética. http://www.fao.org/publications/ es/ (consultado 21 Nov. 2018).

Folgueras, M.; Herrera, L.; Rodríguez, S. y Maza, N. 2010. Identificación del alcance y magnitud de las pudriciones radicales en yuca (M. esculenta Crantz) en diferentes regiones edafoclimáticas de Cuba. Centro Agrícola, vol. 37, no.4, p. 19-24.

Fukuda, W. y Guevara, C. 1998. Descritores morfológicos e agronômicos para a caracterização de mandioca (Manihot esculenta Crantz). 
Cruz das Almas: Embrapa. P.38. Gabriel, L., Streck, N., Uhlmann, L., Silva, M. y Silva, S. 2014. Mudança climática e seus efeitos na cultura da mandioca. Revista Brasileira de Engenharia Agrícola e Ambiental 18: 90-98.

Holguin, P., Collazos, R. y Cock, J. 1984. Fotosíntesis y otros parámetros fisiológicos en plantas de yuca Manihot esculenta Crantz infectadas con virus. Acta Agron.35: 27-38.

João, J., Espinosa, A., Ruiz, Luís, S., González, J. y Rivera, R. 2016. Efectividad de cepas de HMA en el cultivo de la yuca (Manihot esculenta Crantz) en dos tipos de suelos. Cultivos Tropicales, 37(1), 48-56

León, R., Pérez, M., Fuenmayor, F., Gutiérrez, M. y Marín, C. 2014. Caracterización eco-fisiológica de cuatro clones de yuca (Manihot esculenta, Crantz) en el Campo Experimental del INIACENIAP. Agronomía Tropical. 64 (1): 2

León, R., Pérez, M., Fuenmayor, F., Gutiérrez, M., Rodríguez, A., Rodríguez, G. y Marín, C. 2017. Evaluación fisiológica y agronómica de clones promisorios de yuca (Manihot esculenta Crantz) sometidos a condiciones de estrés por sequía. Revista Unellez de Ciencia y Tecnología, 34, 50-57.

Marín, A., Perdomo, D., Alabarrán, J., Fuenmayor, F. y Zambrano, C. 2008. Evaluación agronómica, morfológica y bioquímica de clones élite de yuca a partir de vitroplantas. Interciencia, 33: 365-371.

Mejía , M., El-Sharkawy, M. y Cantillo, S. 1995. Respuesta fotosintética de la yuca (Manihot esculenta Crantz) en dos ambientes. Acta Agron. 1: 41-50.

Mejía, M. 2002. Fisiología de la yuca (Manihot esculenta Crantz). En: B. Ospina, y H. Ceballos, editores, La yuca en el tercer milenio: sistemas modernos de producción, procesamiento, utilización y comercialización. 1a ed. CIAT, COL. p. 34-45. Pastrana, F., Alviz, H. y Salcedo, J. 2015. Respuesta de dos cultivares de yuca a la aplicación de riego en condiciones hídricas diferentes. Acta Agron. 64:48-53.

Ramanujam, T. 1990. Effect of moisture stress on photosynthesis and productivity of cassava. Photosynthetica, 24: 217-224.

Rivera, B., Aceves, L. A., Juárez, J., Palma, D. J., González, R. y González, V. 2012. Zonificación agroecológica y estimación del rendimiento potencial del cultivo de la yuca (Manihot esculenta Crantz) en el estado de Tabasco, México. Avances en investigación agropecuaria, 16(1).

Rodríguez, M. 2013. Incidencia de las variables climáticas en los rendimientos de los cultivos transitorios en la provincia del Alto Magdalena 1992-2013. Tesis magister en ciencias económicas. Universidad Santo Tomas. Bogotá

SAS Institute. 2011. User's guide: Statistics. SAS Inst., Cary, NC.

Serrano, L., Martos, J. y Renedo, V. 2017. Influencia de cuatro sistemas de laboreo en las propiedades físicas de un Fluvisol y en el balance energético en cultivos de raíces y tubérculos. Universidad \&Ciencia, 6(1), 65-81.

Suárez, L. y Mederos, V. 2011. Notes on the cultivation of cassava (Manihot esculenta Crantz). Current trends. Cultivos Tropicales, 32(3), 27-35.

Tezara, W., Mitchell, V., Driscoll, S. y Lawlor, D. 2002. Effects of water deficit and its interaction with $\mathrm{CO} 2$ supply on the biochemistry and physiology of photosynthesis in sunflower. Journal of Experimental Botany, 53: 1781-1791.

Valle, T. 1990. Cruzamentos dialélicos em mandioca (Manihot esculenta Crantz). Tese (Doutorado em Agronomia) - Escola Superior de Agricultura "Luiz de Queiroz" da Universidade 